\title{
Effect of Hyperbaric Pressure During Scuba Diving on Autonomic Modulation of the Cardiac Response: Application of the Continuous Wavelet Transform to the Analysis of Heart Rate Variability
}

\author{
Eduardo Barbosa*; Juan M. García-Manso*; Juan M. Martín-González*; \\ Samuel Sarmiento*; Francisco J. Calderónt; Marzo E. Da Silva-Grigolettoł
}

\begin{abstract}
This study sought to determine the effects of hyperbaric pressure on heart rate modulation, by analyzing potential changes in heart rate variability (HRV). Ten divers were exposed to pressures of 1, 2, 3, and 4 atmospheres absolute (ATA). The test was performed in a hyperbaric chamber. Heart rate (HR) was recorded in supine subjects for 10 minutes per atmosphere. HRV was analyzed in the frequency mode (fast-Fourier transform and continuous wavelet transform). Results confirmed bradycardia as pressure increased. The drop in HR attained statistical significance after 2, 3 , and 4 ATA. Signal energy (normalized TP values) rose progressively, becoming significant at 2 ATA. High frequency and low frequency displayed similar behavior in both cases. Although frequency band peaks did not yield clear results, continuous wave transform analysis showed that the frequency spectrum tended to shift into the high-frequency range as pressure increased. In summary, increased pressure prompted increased bradycardia, and HRV shifted into high-frequency range.
\end{abstract}

\section{INTRODUCTION}

Scuba diving is associated with a number of major functional responses, including bradycardia. Yet although this particular response has been the object of considerable research, ${ }^{1-12}$ the mechanisms prompting the drop in heart rate have yet to be fully clarified. One approach to the study of this phenomenon has involved the use of hyperbaric chambers to simulate the changes in pressure that the body undergoes during immersion. Findings appear to suggest that there is no single mechanism behind this response, but rather that heart rate variability (HRV) is because of the effects of multiple factors on various body systems. For that reason, a number of stimuli have been used to analyze bradycardia during diving; these include increased partial pressure of oxygen at lung level,,${ }^{4,10,13-15}$ changes in temperature, ${ }^{12,16-20}$ and increased ambient pressure..$^{21-23}$

Changes in heart rate presumably affect internal dynamics, and thus the mechanisms by which the autonomic nervous system controls the heart; as a result, the time interval between beats could also be modified by the physical and mechanical changes entailed in diving. ${ }^{11}$ Heart rate variability has become the conventionally accepted term to describe variations of both instantaneous heart rate and R-R interval, ${ }^{24}$ it is a concept used in cardiology for the analysis of the control that the vegetative nervous system exerts on cardiac rhythm modulation. This study sought to examine the way these changes are mani-

*Universidad de Las Palmas de Gran Canaria, 35017 Las Palmas de Gran Canaria, Spain.

$\dagger$ Universidad Politécnica de Madrid, 28040 Madrid, Spain.

$\ddagger$ Andalusian Center of Sports Medicine, Plaza de Vistalegre, 14004 Córdoba, Spain.

The authors report there are no conflicts of interest for this work. fested in subjects exposed to changes in ambient temperature using a hyperbaric chamber.

\section{MATERIALS AND METHODS}

\section{Subjects}

Ten healthy volunteers (nine men, one woman) took part in this study. Mean age was 38.89 years $( \pm 6.33)$; mean weight $72.71 \mathrm{~kg}( \pm 8.64)$, and mean height $1.72 \mathrm{~m} .( \pm 0.05)$. All subjects were frequent divers and were qualified dive masters or instructors. Six subjects also engaged in other forms of physical exercise. All subjects signed a consent form based on the Declaration of Helsinki guidelines (World Medical Association) on research in human beings.

\section{Test Protocol}

All subjects underwent medical checks and morphologicalprofile measurements before the experiment. Those with no experience of hyperbaric chambers were allowed to familiarize themselves beforehand, to avoid adverse reactions during testing that might affect the heart signal. In a single session, supine subjects were exposed to four levels of atmospheric pressure $(1,2,3$, and 4 atmospheres absolute [ATA]) (Fig. 1). The subject was allowed to stabilize between changes of pressure, and heart signals were then recorded for 10 minutes per atmosphere.

\section{Material}

The experiment was performed in a marine surplus supplies 54 inch twin-lock decompression chamber, provided by "Top Dive" diving center. Heart signals were recorded beat by beat using a Polar S810i heart rate monitor (Polar Electro Oy, Finland); this apparatus has been validated against the electrocardiogram (ECG) system in several studies. ${ }^{25,26}$ 


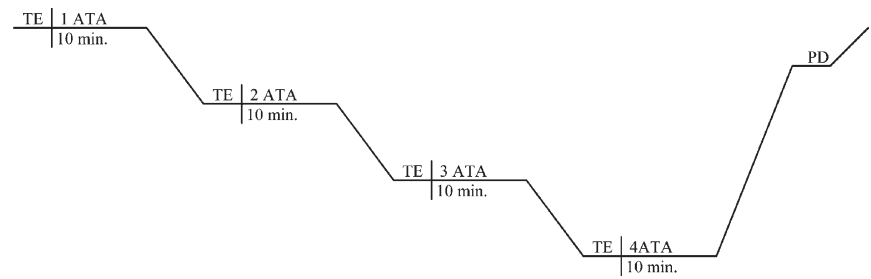

FIGURE 1. Hyperbaric chamber test protocol. ST, HR stabilization time; DS, decompression stopped.

\section{Signal Treatment}

Data sets obtained on the heart rate monitor were treated and analyzed using a commercial software program (MatLab v6.5). After eliminating outliers, a low-pass filter (Db8) was applied for detrending purposes (to remove the very lowfrequency component). Linear interpolation was then used to homogenize the number of data per unit of time. The continuous wavelength transform (CWT) was calculated using the sixth order Morlet wavelet. The CWT spectrogram provides a graphical representation of spectral power at every moment of the test. Signal energy was calculated as the sum of wavelet coefficients at every moment, values being represented in the signal total power (TP) and in each frequency band (high frequency $[\mathrm{HF}]$ and low frequency $[\mathrm{LF}])$.

\section{Statistical Treatment}

The results for time/frequency (wavelet) analysis were accompanied by descriptive statistics, using the mean values (HR, TP, LF and HF) obtained at each atmosphere. Differences between data were determined by a repeated measures ANOVA (Fisher post hoc test); the significance limit was set at $p \leq 0.05$.

\section{RESULTS}

Mild progressive bradycardia was observed as ambient pressure increased (see Figure 2). The reference value was resting HR at an ambient pressure of 1 atmosphere absolute (ATA). The increase in bradycardia reached statistical significance for values of 2, 3, and 4 ATA; this statistical difference was observed both with regard to 1 ATA and among all values (Fig. 2).

By contrast, HRV increased as atmospheric pressure rose. The change in total power with increasing pressure is shown in Figure 3A. When data were expressed as normalized values, changes in total spectral density became significant only for 2, 3, 4 ATA and only with regard to 1 ATA (Fig. 3B). However, this was not the case when data were expressed as absolute values $\left(\mathrm{ms}^{2}\right)$ (Fig. 3A). HF and LF behavior is shown in Figure 3, C-F, respectively.

\section{DISCUSSION}

The results obtained confirmed that increase in ATA and drop in heart rate are related. Similar findings are reported by other authors. ${ }^{27,28}$ Yamazaki et al. ${ }^{29}$ observed a significant decrease in HR at 3 ATA (Nitrox 0.4 atm). In the same study, when atropine and propanolol were used to block extrinsic activity,

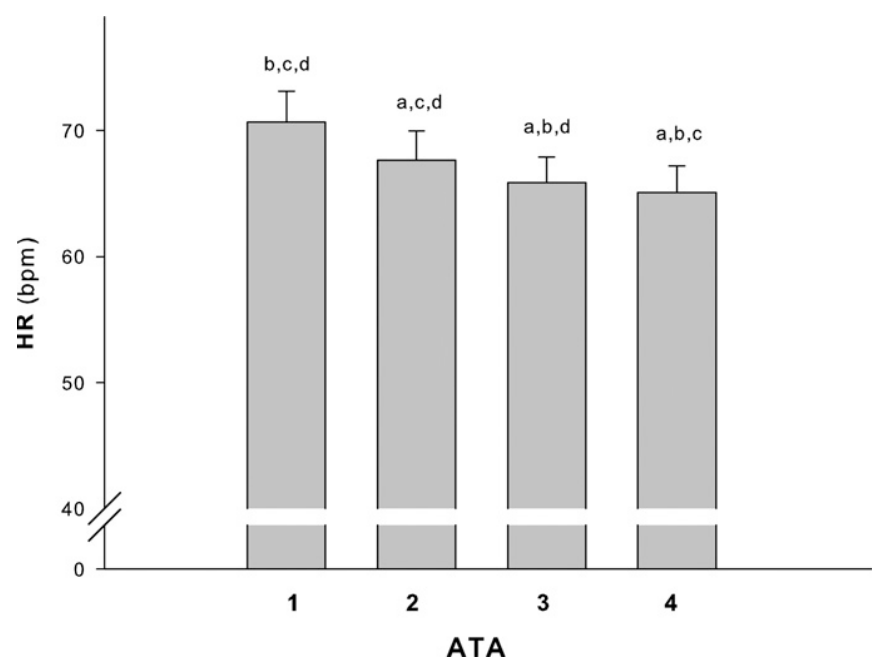

FIGURE 2. Heart rate in bpm. Values are means and SEM. (a) Significant differences $(p<0.05)$ compared to 1 ATA. (b) Significant differences $(p<$ $0.05)$ compared to 2 ATA. (c) Significant differences $(p<0.05)$ compared to 3 ATA. (d) Significant differences $(p<0.05)$ compared to 4 ATA.

no significant differences were found between 1 and 3 ATA. The factors responsible for this bradycardia have yet to be fully clarified.

This phenomenon has traditionally been attributed, among other things, to the activation of facial-skin temperature receptors, which detect the drop in temperature and duly inform cardiovascular control centers, which-via autonomic afferent pathways - cause a decrease in HR. However, studies performed in a hyperbaric chamber are not susceptible to this influence, since increased pressure is matched by increased temperature. Schipke and Pelzer ${ }^{9}$ report no significant differences at a depth of $4 \mathrm{~m}$ when water temperature was maintained constant $\left(27^{\circ} \mathrm{C}\right)$.

It may be assumed, therefore, that the mechanisms prompting bradycardia are multifactorial in origin. Contributory factors may include temperature, changes in volemic status, peripheral vascular resistance, baroreceptor activation, changes in the partial pressure of respiratory gas, and changes in atmospheric pressure. It should be borne in mind in this respect that the human body functions as a complex, selforganizing system.

As ambient pressure increased, lowered heart rate was accompanied by an increase in heart rate variability, i.e., increased variability in the length of the interval between beats. As atmospheric pressure increased, the spectral density of the heart signal analyzed as TP increased exponentially; at 4 ATA, it was $66 \%$ higher than control values (1 ATA). Lund et al. ${ }^{14}$ reported a $104 \%$ increase in TP over baseline at 2.5 ATA, although hyperbaric exposure was much longer than in the present experiment.

Increased HRV was noted over the whole frequency spectrum (HF bands 34\%; LF 77\%). This suggests that parasympathetic tone increases with rising pressure. Although in all cases the spectral signal displayed greater weight in the LF 


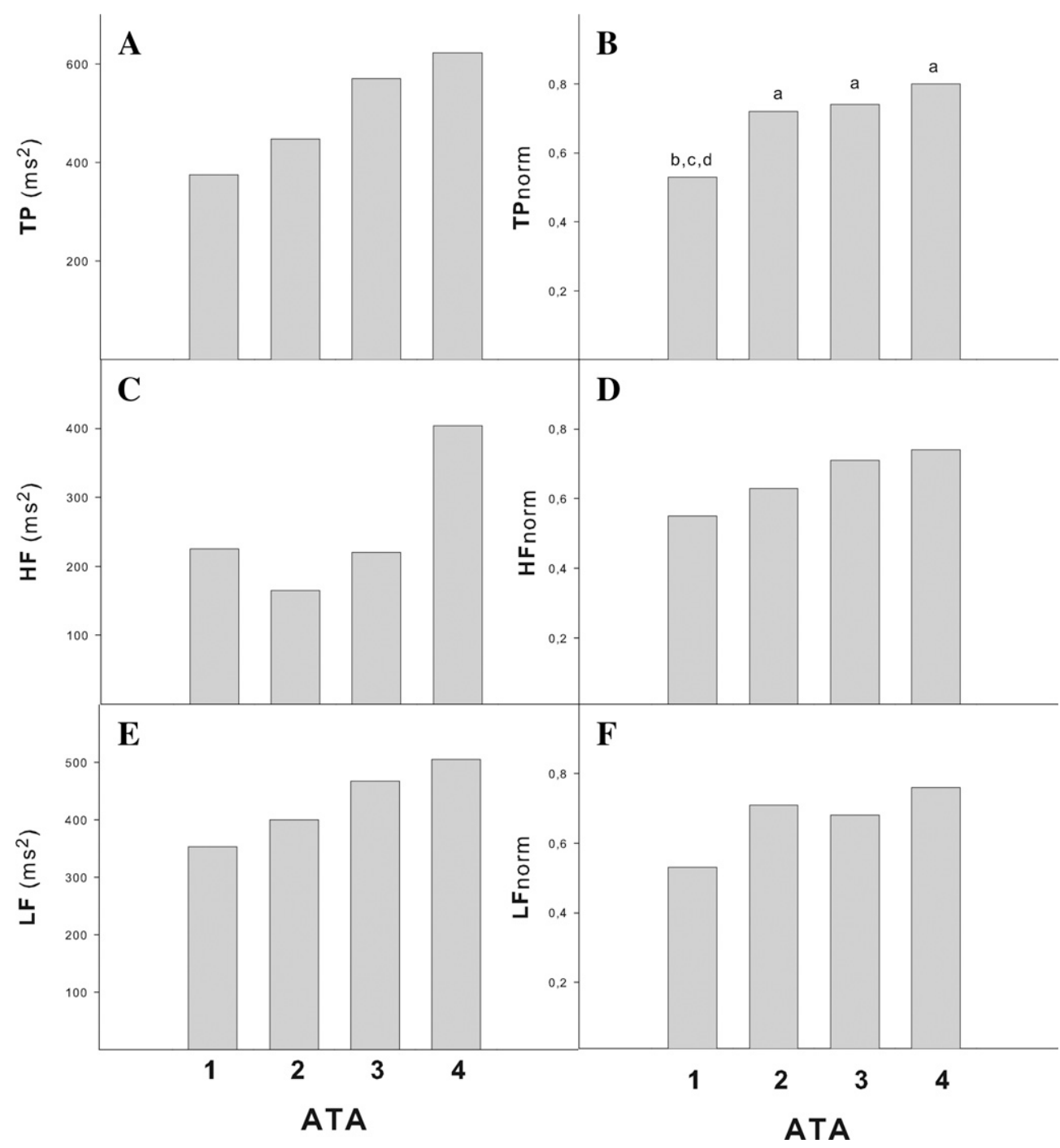

FIGURE 3. Total power (TP), high frequency (HF) and low frequency (LF) absolute and normalized values (value in $x$ ATA $\times$ maximum value $e^{-1}$ ). (A) Significant differences $(p<0.05)$ compared to 1 ATA. (B) Significant differences $(p<0.05)$ compared to 2 ATA. (C) Significant differences $(p<0.05)$ compared to 3 ATA. (D) Significant differences $(p<0.05)$ compared to 4 ATA.
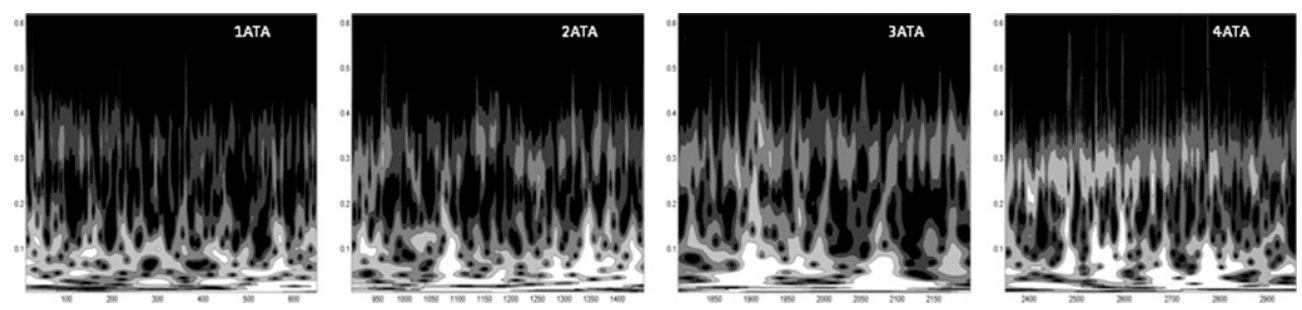

FIGURE 4. Spectrogram (CWT) of a study subject at 1,2,3, and 4 ATA. White indicates greatest density and black, lowest density.

band, the relative weighting changed as atmospheric pressure increased, with a marked shift into the HF range $(24.95 \%$ vs. $30.91 \%$ ). Lund et al. ${ }^{14}$ noted a mean increase in HF power of up to $400 \%$, probably because of prolonged hyperbaric oxygen (HBO). This would suggest greater parasympathetic modulation of the cardiac response as atmospheric pressure increases. Here, however, although the trend was unmistak- able (see example in Figures 4 and 5), differences in spectral density displayed statistical significance only in terms of normalized TP values, a phenomena that may be because of the duration of the test.

Spectral density increased at all frequencies, but the most marked increases were observed in the 0.2 to $0.3 \mathrm{~Hz}$ range. This was particularly interesting since changes in high-frequency 
peaks in an HRV spectrogram are closely related to the respiratory mechanics of the subject (respiratory sinus arrhythmia). Heart rate increases on inhalation and decreases on exhalation. Divers probably adopt a low-frequency breathing pattern, similar to that adopted during immersion, to minimize air intake. In conclusion, an increase in atmospheric pressure is one factor triggering immersion bradycardia. Lowered heart tone in response to increased pressure is accompanied by increased heart rate variability over the whole frequency spectrum and particularly in the HF range.

\section{ACKNOWLEDGMENTS}

This research was supported by the Spanish Ministry of Education, through the Consejo Superior de Deportes.

\section{REFERENCES}

1. Kenmure AC, Murdoch WR, Hutton I, Cameron AJ: Hemodynamic effects of oxygen at 1 and 2 ATA pressure in healthy subjects. J Appl Physiol 1972; 32: 223-6.

2. Ornhagen HC, Hogan PM: Hydrostatic pressure and mammalian cardiacpacemaker function. Undersea Biomed Res 1977; 4(4): 347-58.

3. Torbati D, Parolla D, Lavy S: Organ blood flow, cardiac output, arterial blood pressure and vascular resistance in rats exposed to various oxygen pressures. Aviat Space Environ Med 1979; 50: 256-63.

4. Shida KK, Lin YC: Contribution of environmental factors in development of hyperbaric bradycardia. J Appl Physiol 1981; 50: 731-5.

5. Stur LEB, Bergo GW, Tyssebotn I: Systemic hemodynamics during hyperbaric oxygen exposure in rats. Aviat Space Environ Med 1994; 65: $531-8$.

6. Istepanian RS, Woodward B: Microcontroller-based underwater acoustic ECG telemetry system. IEEE Trans Inf Technol Biomed 1997; 1(2): $150-4$.

7. Il'in VM: The circulatory changes as dependent on the type of autonomic homeostasis in divers in dives to a depth of $65 \mathrm{~m}$. Fiziol Zh 1999; 45(5): $38-48$.

8. Neubauer B, Tetzlaff K, Staschen CM, Bettinghausen E: Cardiac output changes during hyperbaric hyperoxia. Int Arch Occup Environ Health 2001; 74(2): 119-22.

9. Schipke JD, Pelzer M: Effects of immersion, submersion and scuba diving on heart rate variability. Br J Sports Med 2001; 35: 174-80.

10. Lund V, Laine J, Laitio T, Kentala E, Jalonen J, Scheinin H: Instantaneous beat-to-beat variability reflects vagal tone during hyperbaric hyperoxia. Undersea Hyperb Med 2003; 30(1): 29-36.

11. Camporesi EM, Bosco G: Ventilation, gas exchange and exercise under pressure. In: Bennett and Elliott's Physiology and Medicine of Diving, Ed 5, pp 77-114. Edited by Brubbakk AO, Neuman TS. Edinburgh, UK, Saunders, 2003.
12. Kemmer A, Welslau W, Muth CM: Injuries caused by pressure differences while diving. MMW Fortschr Med 2005; 147(27-28): 33-4.

13. Lund V, Kentala E, Scheinin H, Klossner J, Sariola-Heinonen K, Jalonen $\mathrm{J}$ : Heart rate variability in healthy volunteers during normobaric and hyperbaric hyperoxia. Acta Physiol Scand 1999; 167: 29-35.

14. Lund V, Kentala E, Scheinin H, Klossner J, Sariola-Heinonen K, Jalonen J: Hyperbaric oxygen increases parasympathetic activity in professional divers. Acta Physiol Scand 2000; 170(1): 39-44

15. Kurita A: Effects of severe hyperbaric pressure on autonomic nerve functions. Mil Med 2002; 167(11): 934-8.

16. Wallen LL, Rhodes RA, Shulke HR: Physical properties and chemical composition of beta-glucans from fleshy fungi. Appl Microbiol 1965; 13: 272-8.

17. Hochachka PW: Brain, lung, and heart functions during diving and recovery. Science 1981; 212(4494): 509-14.

18. Pelaia P, Rocco M, Tritapepe L, Bortone C: The treatment of arterial gas embolism with hyperbaric oxygenation. Minerva Anestesiol 1992; 58(10): 827-30

19. Stamler A, Wang SY, Aguirre DE, Johnson RG, Sellke FW: Cardiopulmonary bypass alters vasomotor regulation of the skeletal muscle microcirculation. Ann Thorac Surg 1997; 64(2): 460-5.

20. Foster GE, Sheel AW: The human diving response, its function, and its control. Scand J Med Sci Sports 2005; 15: 3-12.

21. Andersen A, Hillestad L: Hemodynamic responses to oxygen breathing and the effect of pharmacological blockade. Acta Med Scand 1970; 188(5): 419-24

22. Sagawa S, Claybaugh JR, Shiraki K, Park YS, Mohri M, Hong SK: Characteristics of increased urine flow during a dry saturation dive at 31 ATA. Undersea Biomed Res 1990; 17(1): 13-22.

23. Molénat F, Boussuges A, Grandfond A, et al: Haemodynamic effects of hyperbaric hyperoxia in healthy volunteers: an echocardiographic and Doppler study. Clin Sci 2004; 106: 389-95.

24. Task force of the European Society of Cardiology and the North American Society of Pacing and Electrophysiology: Heart rate variability: standards of measurement, physiological interpretation and clinical use. Circulation 1996; 93: 1043-65.

25. Gamelin FX, Berthoin S, Bosquet L: Validity of the Polar S810 heart rate monitor to measure R-R intervals at rest. Med Sci Sports Exerc 2006; 38: 887-93.

26. Kingsley M, Lewis MJ, Marson RE: Comparison of Polar 810s and an ambulatory ECG system for RR interval measurement during progressive exercise. Int J Sports Med 2005; 26: 39-44.

27. Yamazaki F, Wada F, Nagaya K, et al: Autonomic mechanisms of bradycardia during nitrox exposure at 3 atmospheres absolute in humans. Aviat Space Environ Med 2003; 74: 643-8.

28. Yamauchi K, Tsutsui Y, Endo Y, Sagawa S, Yamazaki F, Shiraki K: Sympathetic nervous and hemodynamic responses to lower body negative pressure in hyperbaria in men. Am J Physiol Regul Integr Comp Physiol 2002; 282: R38-45.

29. Yamazaki F, Shiraki K, Sagawa S: Assessment of cardiac autonomic nervous activities during heliox exposure at 24 atm abs. Aviat Space Environ Med 1998; 69: 643-6. 